\title{
An Upstream Flux Splitting Method for Hydrodynamic Modeling of Deep Submicron Devices
}

\author{
MIN SHEN ${ }^{\mathrm{a}}$, WAI-KAY YIP ${ }^{\mathrm{b}}$, MING-C. CHENG ${ }^{\mathrm{c}, *}$ and J. J. LIOU ${ }^{\mathrm{d}}$ \\ ${ }^{\mathrm{a}}$ Advanced Materials Research Institute; ${ }^{\mathrm{b}}$ Department of Electrical Engineering, \\ University of New Orleans, New Orleans, LA 70148, USA; ${ }^{\mathrm{c}}$ Dept. of Electrical \\ and Computer Engineering, Clarkson University, Box 5720, Potsdam, New York 13699-5720 \\ ${ }^{\mathrm{d}}$ Department of Electrical and Computer Engineering, University of Central Florida, \\ Orlando, FL 32816
}

\begin{abstract}
The advective upstream splitting method (AUSM) developed for fluid dynamics problems has been applied to solving hydrodynamic semiconductor equations coupled with the Poisson's equation. In the AUSM, the flux vectors of a fluid system are split into a convective component and a diffusive pressure component. Discretization of these two physically distinct fluxes is thus performed separately in AUSM. Application of the developed hydrodynamic AUSM to a GaAs MESFET with a gate length of $0.1 \mu \mathrm{m}$ has demonstrated its simplicity, efficiency and effectiveness in dealing with the highly nonlinear hydrodynamic device system.
\end{abstract}

Keywords: Device simulation; Hydrodynamic model; Upwind difference; Convective flux

\section{INTRODUCTION}

Many upwind flux splitting schemes have been developed to solve Euler and Navier-Stokes equations $[1-4]$. These approaches are usually classified as flux vector splitting (FVS) or flux difference splitting (FDS). Among these schemes, the FVS approaches, such as Steger-Warming splitting (SWS) [1] and Van Leer splitting (VLS) [2], appear to be simpler and more efficient but the accuracy is limited due to numerical diffusion. On the other hand, the FDS approaches, such as Roe splitting (RS) [3] and Osher splitting (OS) [4] are more accurate. Formulation using FDS is however more complicated, and the computational cost for FDS is considerably higher. Recently, the advective upstream splitting method (AUSM) [5], which combines the features of FYS and FDS approaches, has been developed to achieve the efficiency and simplicity of FVS and the accuracy of FDS. The AUSM has been demonstrated to be robust and efficient for a wide variety of flow problems [5-7].

In this study, we apply the AUSM to the hydrodynamic semiconductor equations coupled with the Poisson's equation to examine its capability for simulation of deep submicron devices. The

\footnotetext{
*Corresponding author: Tel.: 1-315-268-7735, Fax: 315-268-7600, e-mail: mcheng@clarkson.edu
} 
numerical flux in the AUSM is separated into the convective and pressure-like contributions at the cell interface. The convective flux is carried by the carrier average velocity but the pressure-like flux is governed by the carrier random velocity. Discretization of these two physically distinct fluxes is thus performed separately in AUSM.

\section{HYDRODYNAMIC EQUATIONS FOR SEMICONDUCTOR DEVICES}

The hydrodynamic equations [8-12] for charge carriers in semiconductor devices include conservation equations for carrier density $n$, momentum $\mathbf{p}$ and energy $w$, which are

$$
\begin{gathered}
\frac{\partial n}{\partial t}+\nabla \cdot(n \mathbf{v})=0 \\
\frac{\partial n \mathbf{p}}{\partial t}+\nabla \cdot n \mathbf{p} \mathbf{v}+\nabla n k_{B} T_{e}=-e n \mathbf{E}-\frac{n \mathbf{p}}{\tau_{p}} \\
\frac{\partial n w}{\partial t}+\nabla \cdot n w \mathbf{v}+\nabla \cdot n k_{B} T_{e} \mathbf{v}+\nabla \cdot n \mathbf{Q} \\
=-e n \mathbf{E} \cdot \mathbf{v}-\frac{n\left(w-w_{0}\right)}{\tau_{w}}
\end{gathered}
$$

where $\mathbf{p}=\mathrm{m}^{*} \mathbf{v}, \mathbf{v}$ is the average velocity of carriers, $m^{*}$ effective mass, $k_{B}$ the Boltzmann constant, $q$ the elementary charge, $\mathbf{E}$ the applied electric field, $\tau_{p}$, the momentum relaxation time, $\tau_{w}$ the energy relaxation time, and $w_{0}$ the thermal energy at lattice temperature. The coefficients $\tau_{m}, \tau_{m}$, and $m^{*}$ are assumed energy dependent and extracted from Monte Carlo simulation. $\mathbf{Q}$ is the heat flux and described by the gradient of electron temperature $T_{e}$ :

$$
\mathbf{Q}=-\kappa \nabla T_{e} \quad \text { and } \quad \kappa=\frac{5}{2} \frac{k_{B}^{2} T_{e} \tau_{p}}{m^{*}}
$$

where $\kappa$ is the thermal conductivity. The mean energy $w$ is related to $T_{e}$ and $\mathbf{v}$ by

$$
w=\frac{3}{2} k_{B} T_{e}+\frac{1}{2} m^{*} \mathbf{v}^{2} .
$$

The electric field $\mathbf{E}$ is obtained from the potential $\psi$ which is solved from Poisson's equation:

$$
\mathbf{E}=-\nabla \psi, \quad \text { and } \quad \nabla^{2} \psi=-\frac{q\left(N_{d}-n\right)}{\varepsilon_{s}},
$$

where $\varepsilon_{s}$ is the dielectric constant of the semiconductor and $N_{d}$ is the n-type impurity concentration. In this study, only the $n$-type impurity.

The hydrodynamic model described by Eqs. (1) - (3) and the Poisson's equation in Eq. (4) are solved self-consistently. The AUSM is used to obtain the solution of the hydrodynamic equations, and central difference is employed to determine the potential from Poisson's equation. It should be noted that the hydrodynamic model used to demonstrate the capability of the upstream flux splitting method is a semiclassic approach. For ultra- small devices where quantum phenomena have strong influences on device characteristics, quantum corrections are required.

\section{FORMULATION \\ FOR THE ADVECTIVE UPSTREAM SPLITTING METHOD}

The hydrodynamic equations given in Eqs. (1a)(1c) can be expressed by a single equation in vector form. In a $2 \mathrm{D}$ device, this vector equation is written as

$$
\frac{\partial \mathbf{U}}{\partial t}+\frac{\partial \mathbf{F}}{\partial x}+\frac{\partial \mathbf{G}}{\partial y}=\mathbf{H}
$$

where $\mathbf{U}$ is the vector of independent variables and chosen to be

$$
\mathbf{U}=\left[\begin{array}{lll}
n n p_{x} & n p_{y} & n w
\end{array}\right]^{T},
$$

the flux vector in the $x$ direction is given as

$$
\mathbf{F}=\left[n v_{x} n\left(p_{x} v_{x}+k_{B} T_{e}\right) n p_{y} v_{x} n v_{x}\left(w+k_{B} T_{e}\right)\right]^{T},
$$


the flux vector in the $y$ direction is expressed as

$$
\mathbf{G}=\left[n v_{y} n p_{x} v_{y} n\left(p_{y} v_{y}+k_{B} T_{e}\right) n v_{y}\left(w+k_{B} T_{e}\right)\right]^{T},
$$

and the source vector becomes

$$
\mathbf{H}=\left[\begin{array}{c}
0 \\
-n q E_{x}-\frac{n p_{x}}{\tau_{p}} \\
-n q E_{y}-\frac{n p_{y}}{\tau_{p}} \\
-q n E_{x} v_{x}-q E_{y} v_{y}-\frac{\partial\left(n Q_{x}\right)}{\partial x}-\frac{\partial\left(n Q_{y}\right)}{\partial y}-\frac{n\left(w-w_{o}\right)}{\tau_{w}}
\end{array}\right]
$$

The fluxes given in Eqs. (6b) and (6c) can be decomposed into convective and pressure-like fluxes:

$$
\begin{gathered}
\mathbf{F}=\mathbf{F}^{c}+\mathbf{F}^{p}=\left[\begin{array}{c}
n \\
n p_{x} \\
n p_{y} \\
n\left(w+k_{B} T_{e}\right)
\end{array}\right] v_{x}+\left[\begin{array}{c}
0 \\
k_{B} T_{e} \\
0 \\
0
\end{array}\right], \\
\mathbf{G}=\mathbf{G}^{c}+\mathbf{G}^{p}=\left[\begin{array}{c}
n \\
n p_{x} \\
n p_{y} \\
n\left(w+k_{B} T_{e}\right)
\end{array}\right] v_{y}+\left[\begin{array}{c}
0 \\
k_{B} T_{e} \\
0 \\
0
\end{array}\right],
\end{gathered}
$$

The convective fluxes, $\mathrm{F}^{c}$ and $\mathrm{G}^{c}$, are carried by the carrier average velocities, $v_{x}$ and $v_{y}$, respectively. The pressure-like fluxes, $\mathrm{F}^{p}$ and $\mathrm{G}^{p}$, are governed by the carrier random velocity. The random velocity can be show to be $c=$ $\sqrt{5 k_{B} T_{e} / 3 m^{*}}$ (analogy to the sound velocity in fluid dynamics). As proposed in [5], these two fluxes are discretized differently and weighted by the Mach numbers. Evaluation of the fluxes at the interface of the adjacent cells is described below.

\subsection{Convective Fluxes}

The modified Mach numbers, $M_{x 1 / 2}$ and $M_{y 1 / 2}$, at the interface are given as

$$
\begin{aligned}
& M_{x 1 / 2}=M_{x L}^{+}+M_{x R}^{-} \text {and } \\
& M_{y 1 / 2}=M_{y D}^{+}+M_{y U}^{-},
\end{aligned}
$$

where subscripts $L$ and $R$ denote the parameter values taken at the grid point to the left and to the right of the interface, respectively. The split Mach numbers, $M_{x}^{+}$and $M_{x}^{-}$, are defined as

$$
M_{x, y}^{+}=\left\{\begin{array}{cl} 
\pm \frac{1}{4}\left(M_{x, y} \pm 1\right)^{2} & \text { if }\left|M_{x, y}\right| \leq 1 \\
\frac{1}{2}\left(M_{x, y} \pm\left|M_{x, y}\right|\right) & \text { if }\left|M_{x, y}\right|>1
\end{array}\right.
$$

where Mach numbers are $M_{x}=v_{x} / c$ and $M_{y}=v_{y} / c$. Using the modified Mach numbers, the convective flux $\mathbf{F}^{c}$ at the interface of two adjacent grid points in the $x$ direction is given as

$$
\begin{aligned}
F_{1 / 2}^{c}=v_{x 1 / 2}\left[\begin{array}{c}
n \\
n p_{x} \\
n p_{y} \\
n\left(w+k_{B} T_{e}\right)
\end{array}\right]_{L / R} \\
=M_{x 1 / 2}\left[\begin{array}{c}
c n \\
c n p_{x} \\
c n p_{y} \\
c n\left(w+k_{B} T_{e}\right)
\end{array}\right]_{L / R},
\end{aligned}
$$

where the flux at the grid point left to the interface is taken for $M_{x 1 / 2} \geq 0$ and right to the interface for $M_{x 1 / 2}<0$. Similarly $\mathbf{G}^{c}$ at the interface of two adjacent grid points in they direction is given as

$$
\begin{aligned}
\mathbf{G}_{1 / 2}^{c} & =v_{y 1 / 2}\left[\begin{array}{c}
n \\
n p_{x} \\
n p_{y} \\
n\left(w+k_{B} T_{e}\right)
\end{array}\right]_{D / U}, \\
& =M_{x 1 / 2}\left[\begin{array}{c}
c n \\
c n p_{x} \\
c n p_{y} \\
c n\left(w+k_{B} T_{e}\right)
\end{array}\right]_{D / U}
\end{aligned}
$$

where the flux at the grid point below the interface is taken for $M_{y 1 / 2} \geq 0$ and above the interface for $M_{y 1 / 2}<0$. 


\subsection{Pressure-like Fluxes}

The pressure-like fluxes are first split as follows:

$$
\begin{aligned}
& \mathbf{F}^{\mathrm{P} \pm}=\left\{\begin{array}{c}
\frac{\mathrm{F}^{\mathrm{P}}}{2}\left(1 \pm M_{x}\right) \text { if } M_{x} \leq 1, \\
\frac{\mathrm{F}^{\mathrm{P}}}{2}\left(M_{x} \pm\left|M_{x}\right|\right) / M_{x} \text { if } M_{x}>1,
\end{array}\right. \text { and } \\
& \mathbf{G}^{\mathrm{P} \pm}=\left\{\begin{array}{c}
\frac{\mathrm{G}^{\mathrm{P}}}{2}\left(1 \pm M_{y}\right) \text { if } M_{y} \leq 1, \\
\frac{\mathbf{G}^{\mathrm{P}}}{2}\left(M_{y} \pm\left|M_{y}\right|\right) / M_{y} \text { if } M_{y}>1,
\end{array}\right.
\end{aligned}
$$

The fluxes at the interface of the adjacent cells are taken as

$$
\mathbf{F}_{1 / 2}^{p}=\mathbf{F}_{L}^{p+}+\mathbf{F}_{R}^{p-} \quad \text { and } \quad \mathbf{G}_{1 / 2}^{p}=\mathbf{G}_{L}^{p+}+\mathbf{G}_{R}^{p-}
$$

Using Eqs. (10a) and (10b), upwind difference is weighted by the modified Mach numbers, $M_{x 1 / 2}$ and $M_{y 1 / 2}$, for the convective fluxes at the interface of adjacent cells in the discretized central-difference equation for Eq. (5). On the other hand, central difference weighted by $M_{x}$ and $M_{y}$ appears in the pressure-like fluxes.

\section{NUMERICAL SIMULATION AND DISCUSSION}

Simulations using the AUSM have been performed on an $n$-channel GaAs MESFET with a gate length of $0.1 \mu \mathrm{m}$ at room temperature. The detailed device structure is given in Figure 1(a). The current density vectors in this $2 \mathrm{D}$ structure at $V_{g s}=-0.8 \mathrm{~V}$ and $V_{d s}=1.5 \mathrm{~V}$ are also shown in Figure 1(b) where the current flow and the depletion region in the device can be clearly observed.

The electron concentration and temperature are illustrated in Figures 2(a) and 2(b). Due to the reverse-biased Schottky barrier under the gate, the channel is form on the bottom of the device as shown in Figure 1(b) and Figure 2(a). The

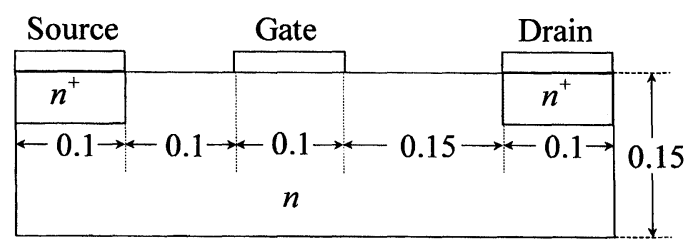

(a)

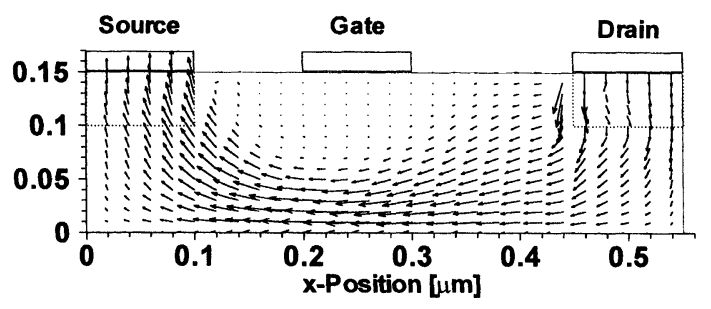

(b)

FIGURE 1 (a) The 2D depletion-model GaAs $n$-channel MESFET structure used in the hydrodynamic simulation. Source. Gate, and drain metal contacts are shown on the top of the device. $N_{d}=10^{17} \mathrm{~cm}^{-3}$ in the $n$ region, and $N_{d}=$ $3 \times 10^{17} \mathrm{~cm}^{-3}$ in the $n^{+}$source/drain. (b) The current density vectors at $V_{d s}=1.5 \mathrm{~V}$ and $V_{g s}=-0.8 \mathrm{~V}$, where the vector length is proportional to the magnitude of the current density vector.

reverse-biased Schottky barrier and applied drainsource voltage are also responsible for the extremely large density gradient near the drain metal edge at $(x, y)=(0.45 \mu \mathrm{m}, 0.15 \mu \mathrm{m})$. Similar discontinuity for electron temperature at some metal edges is also observed in Figure 2(b), particularly near the gate metal edges. This is because the lattice temperature is taken on the metal boundary and zero $E_{x}$ is set on the metal boundary which causes the discontinuity of the tangential component of the electric field $\left(E_{x}\right)$ near the gate metal edges. As shown in Figure 3(a), $E_{x}=0$ along the metal boundary, and extremely strong electric fields (as high as $8 \times 10^{7} \mathrm{~V} / \mathrm{m}$ ) are found at metal edges near $x=0.2$ and $0.3 \mu \mathrm{m}$. Strong field normal to the gate $\left(E_{y}\right)$ under the gate is also shown due to the reverse-biased Schottky barrier. Because of the zero normal component of electric field on the non-metal boundary, discontinuity for 14 is also observed at the metal near $x=0.2$ and $0.3 \mu \mathrm{m}$.

The AUSM appears to be very efficient for hydrodynamic simulation of the GaAs MESFET. 

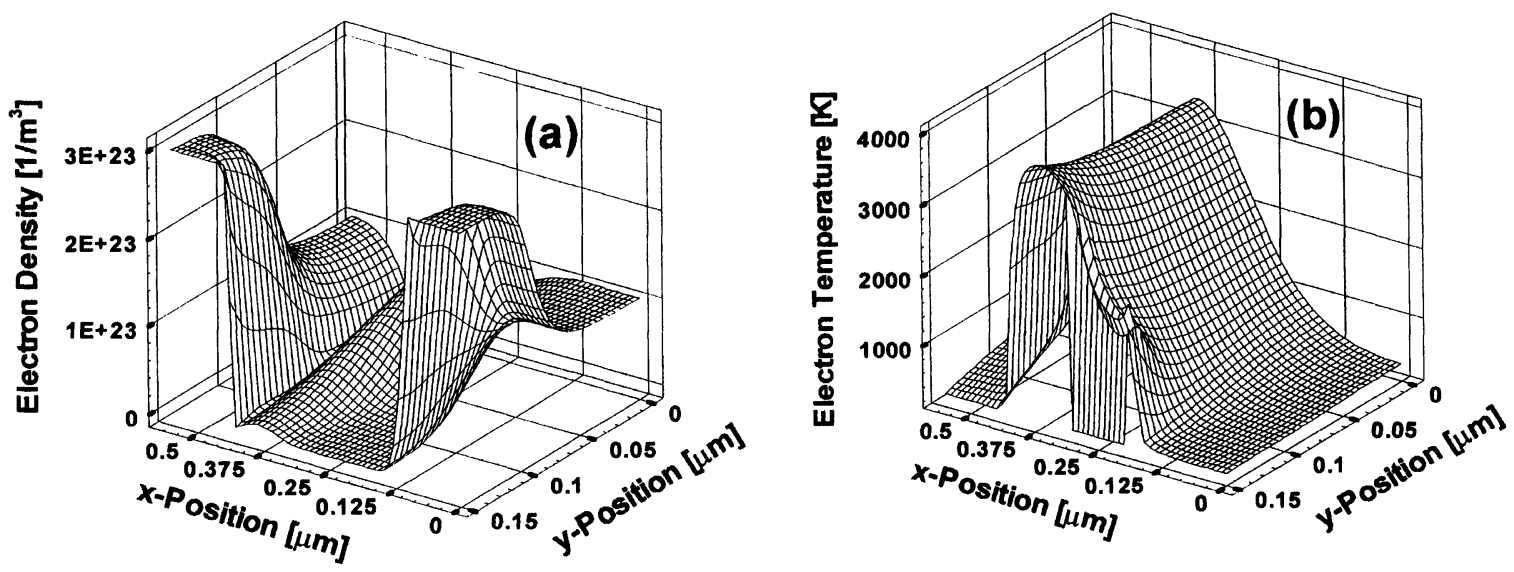

FIGURE 2 (a) Electron density and (b) electron temperature in the MESFET at $V_{d s}=1.5 \mathrm{~V}$ and $V_{g s}=-0.8$.
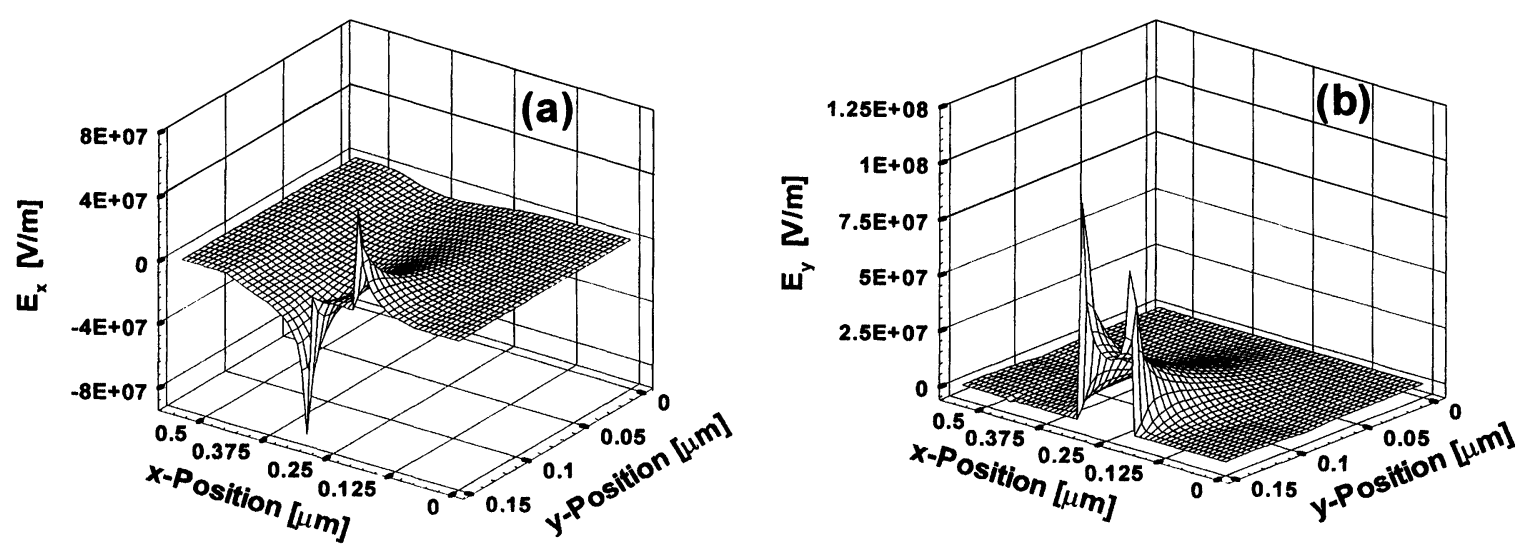

FIGURE 3 (a) $x$-component and (b) $y$-component electric fields in the MESFET at $V_{d s}=1.5 \mathrm{~V}$ and $V_{g s}=-0.8$.

It requires approximately 35 minutes on a Digital Alpha $533 \mathrm{MHz}$ workstation. This study shows that regardless of high gradients of many variables, stable and smooth numerical results can be reached. It demonstrates the effectiveness and efficiency of the AUSM in handling the convective flux and the extremely high-gradient situations.

\section{CONCLUSION}

A hydrodynamic AUSM has been developed and applied to obtain self-consistent solution from hydrodynamic equations and Poisson's equation for electrons in a 2D GaAs MESFET with a gate length of $0.1 \mu \mathrm{m}$. The flux splitting schemes proposed in the AUSM [5] are easy to formulate, and the application to the hydrodynamic device simulation appears to be very efficient. Regardless of high gradients of the variables, the AUSM is able to provide stable and smooth numerical solution. The method has been shown to be very efficient and effective in handling convective and pressure-like fluxes in the highly nonlinear hydrodynamic semiconductor equations in a very small GaAs device. 


\section{Acknowledgement}

The authors Cheng and Liou are partially supported by SRC under Grant Number 2000RJ-873G.

\section{References}

[1] Stegger, J. L. and Warming, R. F. (1981). J. Comput. Phys., 40, 263.

[2] van Leer, B. (1982). Lecture Notes in Physics, 170, Springer-Verlag, New Year/Berlin, 507.

[3] Roe, P. L. (1981). J. Comput. Phys., 43, 357.
[4] Osjer, S. (1981). Math. Studies, 47, North-Holland, Amsterdam, 179.

[5] Meng-Sing Liou and Christopher J. Steffen, Jr. (1993). J. Comput. Phys., 107, 23.

[6] Meng-Sing Liou (1996). J. Comput. Phys., 129, 364.

[7] Richard W. Smith (1999). J. Comput. Phys., 150, 268.

[8] Ming-C. Cheng Liangying Guo, Robert Fithen and Yansheng Luo (1997). J. Phys. D: Appl. Phys., 30, 2343.

[9] Carl L. Gardner (1991). IEEE Trans. Electron Dev., 38, 392.

[10] Benvenuti, A., Coughran, W. M. Jr. and Pinto, M. R. (1997). IEEE Trans. Elect. Dev., 44, 1349.

[11] Smith, A. W. and Brennan, K. F. (1996). Solid St. Elect., 39, 1055.

[12] Melih Ozaydin and Lester F. Estman (1996). Solid St. Elect., 39, 731. 

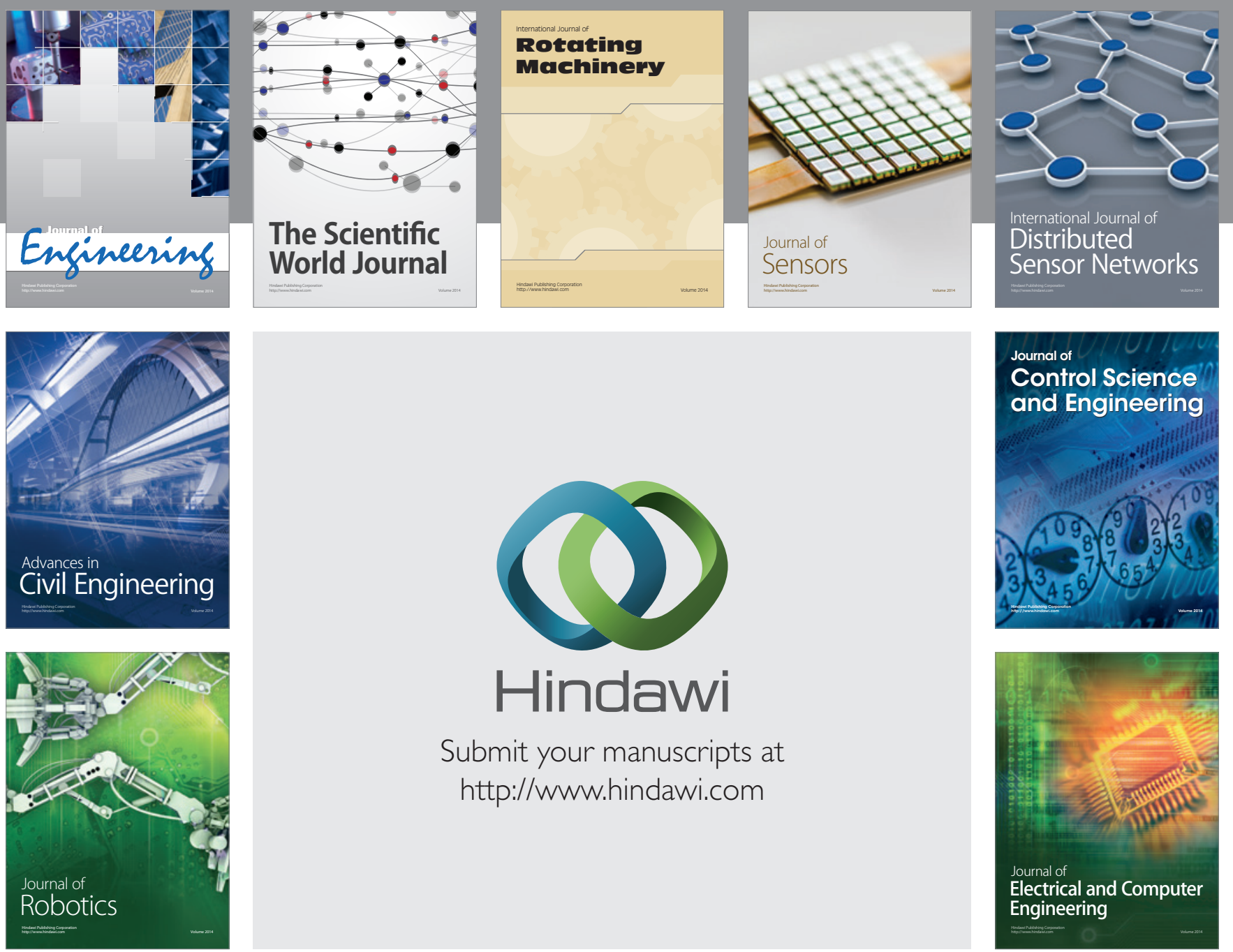

Submit your manuscripts at

http://www.hindawi.com
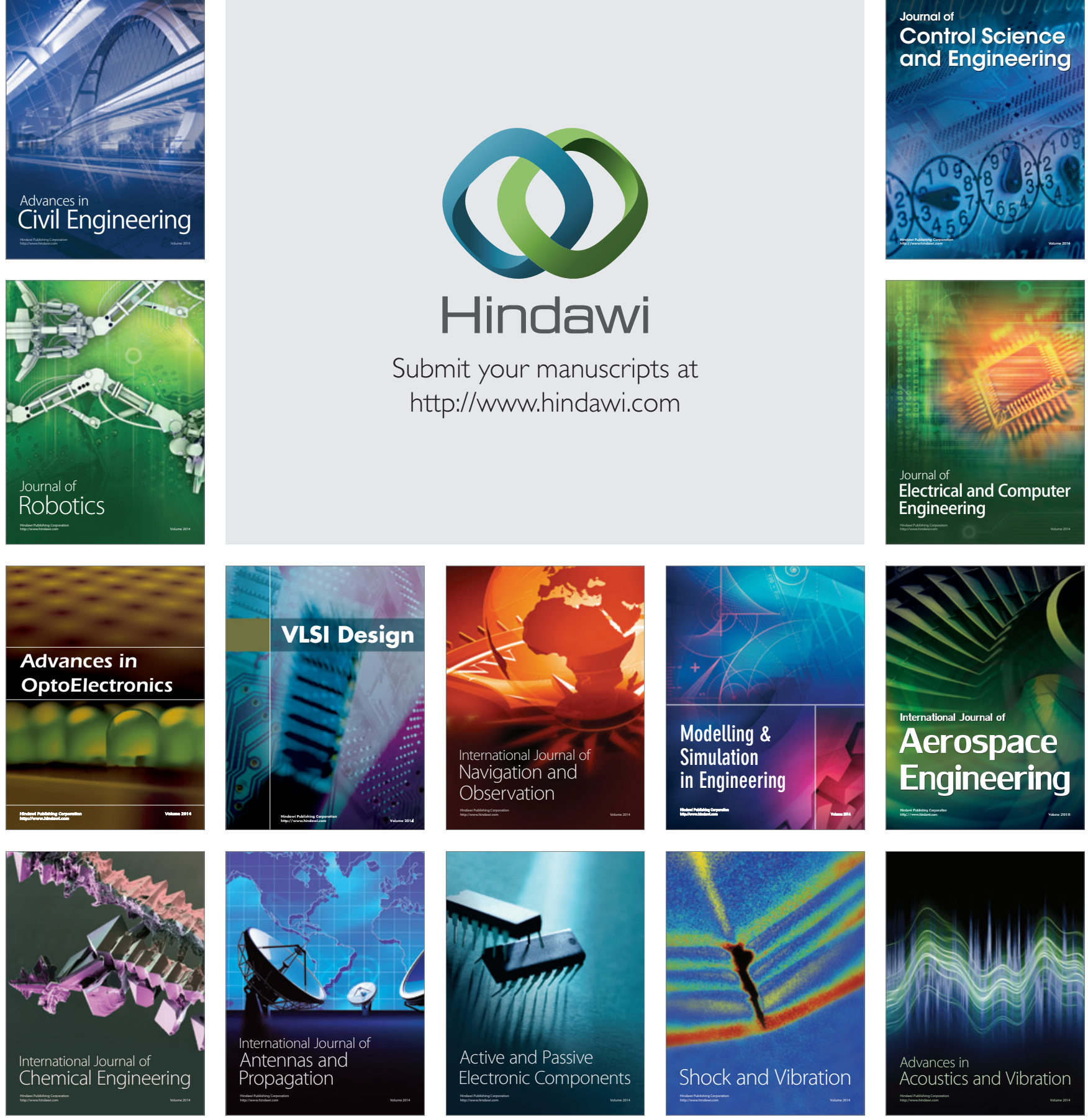